Gut and Liver, Vol. 11, No. 5, September 2017, pp. 728-732

\title{
Assessment of the Risk of Colorectal Cancer Survivors Developing a Second Primary Pancreatic Cancer
}

\author{
Joo Won Chung ${ }^{1,2}$, Moon Jae Chung ${ }^{3}$, Seungmin Bang ${ }^{3}$, Seung Woo Park ${ }^{3}$, Si Young Song ${ }^{3}$, Jae Bock Chung ${ }^{3}$, and \\ Jeong Youp Park ${ }^{3}$ \\ ${ }^{1}$ Division of Gastroenterology, Department of Internal Medicine, National Medical Center, ${ }^{2}$ Department of Medicine, Graduate School, Yonsei \\ University College of Medicine, and ${ }^{3}$ Division of Gastroenterology, Department of Internal Medicine, Institute of Gastroenterology, Yonsei \\ University College of Medicine, Seoul, Korea
}

Background/Aims: We aimed to investigate the incidence of second primary pancreatic cancer (PC) after colorectal cancer (CRC) and to identify risk factors associated with subsequent PC. Methods: The observed incidence of a subsequent $\mathrm{PC}$ in patients with $\mathrm{CRC}$ was standardized using a population with CRC from the Korean Central Cancer Registry (KCCR). The expected incidence rate of PC was obtained by assuming that the select group experienced the same cancer incidence as the corresponding general population in the KCCR. Results: The registry included 4,822 patients with CRC aged 45 to 74 years, representing $16,725.1$ person-years of follow-up. Thirteen patients $(0.3 \%)$ were diagnosed with a subsequent $\mathrm{PC}$, and the overall age-adjusted incidence of second primary PC was 269.6 per 100,000 cases. In contrast, the overall incidence of primary PC in the general population was 18.68 per 100,000 individuals. The standardized incidence ratio of subsequent PC was 14.44 , which was significantly higher in patients with CRC than in the general population. Sex, diabetes mellitus, smoking, body mass index, and a history of receiving chemotherapy as a treatment for $\mathrm{CRC}$ did not increase the risk of subsequent development of PC. Conclusions: The risk of a second primary PC was higher in patients with CRC. Further studies are needed to identify the risk factors and generate a screening strategy for cancer survivors. (Gut Liver 2017;11:728-732)

Key Words: Pancreatic neoplasms; Colorectal neoplasms; Second primary neoplasm

\section{INTRODUCTION}

Due to advances in diagnostic and therapeutic techniques, life expectancies for most cancers have increased over the past decades. However, despite medical progress, the prognosis of pancreatic cancer (PC) is still not favorable, and the 5-year survival rate has only shown a small increase. ${ }^{1}$ Merely $10 \%$ of PC patients are candidates for curative resection at the time of initial diagnosis, without any effective screening method available for early diagnosis of PC. Although intensive surveillance with endoscopic ultrasound for high-risk patients with familial PC could detect PC at an early stage, the cost-effectiveness of such screening method is questionable. ${ }^{2}$ To make matters worse, there are only a few chemotherapy options available for locally advanced, metastatic, or recurred PC. Despite recent positive results with combination chemotherapies, such as FOLFIRINOX ${ }^{3}$ and nab-paclitaxel/gemcitabine, ${ }^{4}$ very few patients get these benefits due to poor Eastern Cooperative Oncology Group (ECOG) performance status and chemo-toxicities. In this regard, early diagnosis of PC and selection of high-risk patients are extremely important.

In general, it is assumed that a patient who previously had cancer (a cancer survivor) is at risk of developing new cancer in another organ. ${ }^{5}$ In particular, cancer survivors for certain gastrointestinal malignancies with long overall survival time such as gastric cancer or colorectal cancer (CRC) may be at a higher risk of a second primary cancer. Investigation of second primary PC can provide clues to etiologic factors, and also identify patients who need more intensive medical surveillance. In this study, we assessed the incidence of second primary PC in patients with CRC compared to the incidence of PC in the general population, and investigated the risk factors for second primary PC.

\footnotetext{
Correspondence to: Jeong Youp Park

Division of Gastroenterology, Department of Internal Medicine, Institute of Gastroenterology, Yonsei University College of Medicine, 50-1 Yonseiro, Seodaemun-gu, Seoul 03722, Korea

Tel: +82-2-2228-1982, Fax: +82-2-2227-7900, E-mail: sensass@yuhs.ac

Received on October 29, 2016. Revised on January 20, 2017. Accepted on January 26, 2017. Published online July 28, 2017 pISSN 1976-2283 eISSN 2005-1212 https://doi.org/10.5009/gnl16526

@ This is an Open Access article distributed under the terms of the Creative Commons Attribution Non-Commercial License (http://creativecommons.org/licenses/by-nc/4.0) which permits unrestricted non-commercial use, distribution, and reproduction in any medium, provided the original work is properly cited.
} 


\section{MATERIALS AND METHODS}

\section{Patients}

From January 2001 to July 2009, we identified consecutive patients with diagnosis of CRC who were prospectively enrolled in the cancer registry database at Severance Hospital in Seoul, Korea. Clinical data were collected for the patients' age, sex, comorbidities (diabetes mellitus [DM], body mass index [BMI] for obesity), and smoking history at the time of initial CRC diagnosis, as well as their chemotherapy history of CRC and the occurrence of second primary cancer during follow-up period. The diagnosis of malignancy in this registry was confirmed histologically. Follow-up data of these patients were collected until August 2011. We selected patients of ages between 45 and 74 years. Follow-up duration was expressed as person-years of observation, and the risk of second primary PC was stratified by 10-year age groups and sex. The Institutional Review Board of Severance Hospital at Yonsei University approved this retrospective study.

\section{Statistics}

The observed incidence rate of second primary PC in CRC patients was standardized using a CRC population in the Korean Central Cancer Registry (KCCR) of the National Cancer Center. ${ }^{6}$ The expected incidence rate of PC was obtained by assuming that these persons experienced the same cancer incidence as prevailed in the corresponding general population in the KCCR. The standardized incidence ratio (SIR) was calculated as the ratio of the observed number to the expected number of PC cases within the age and sex groups. Statistical significance was assessed under the assumption that the observed number of PC followed a Poisson distribution, as the occurrence of second primary PC was relatively rare. ${ }^{7}$

A 95\% confidence interval (CI) was calculated for each SIR using the Fisher exact test. To identify risk factors of PC, potential association of clinical factors with PC was evaluated using either the Pearson chi-square test or the Fisher exact test. To identify independent factors associated with development of a second primary PC, multivariate logistic regression analysis was performed. Adjusted odds ratios were calculated to measure the degree of association, while the Hosmer-Lemeshow test for goodness of fit was used to ensure that the calibration of multivariate analysis was significant for the development of PC. A pvalue less than 0.05 was considered significant, and statistical tests were two-sided. All statistical analyses were performed using SPSS version 18.0 for Windows (SPSS Inc., Chicago, IL, USA).

\section{RESULTS}

A total of 5,988 CRC patients were identified in the registry at our hospital, out of which 4,822 patients were eligible for this
Table 1. Demographic Characteristics of Patients with Colorectal Cancer

\begin{tabular}{lc}
\hline \multicolumn{1}{c}{ Variable } & Value \\
\hline Total patients & 4,822 \\
Male:female & $2,981(61.8): 1,841(38.2)$ \\
Age at initial diagnosis, yr & $61.0(45.0-74.0)$ \\
Age group, yr* & \\
$\quad 45-54$ & $1,153(23.9)$ \\
$55-64$ & $1,962(40.7)$ \\
65-74 & $1,707(35.4)$ \\
Follow-up duration, mo & $40.1(0-384.3)$ \\
Total follow-up duration, person-year & $16,725.09$ \\
Body mass index $\geq 25 \mathrm{~kg} / \mathrm{m}^{2}$ & $1,289(26.7)$ \\
Diabetes mellitus & $670(13.9)$ \\
Smoking history & \\
$\quad$ Smoker or ex-smoker & $922(19.1)$ \\
Never smoker & $3,813(79.1)$ \\
Unknown & $87(1.8)$ \\
Chemotherapy for colorectal cancer & $3,543(73.5)$ \\
\hline
\end{tabular}

Data are presented as number (\%) or median (range).

*The age at the diagnosis of colorectal cancer was divided into 10year groups.

study. We excluded two patients who were diagnosed with PC, in concurrence with the initial diagnostic work up for CRC. The median age at initial diagnosis of CRC was 61.0 years (range, 45.0 to 74.0 years), and $61.8 \%$ of patients were men. The median follow-up duration from CRC diagnosis was 40.1 months (range, 0 to 384.3 months). About one-fifth of CRC patients (19.1\%) had a history of smoking, while three-fourths of them (73.5\%) had received one or more chemotherapies. Patient characteristics of this study are summarized in Table 1 .

Over a period of 16,725.09 person-years of observation, 13 cases of PC (0.3\%) developed in 4,822 CRC patients. The median age reported at initial CRC diagnosis was higher in patients with subsequent PC than those without PC $(\mathrm{p}=0.128)$. The median time interval between diagnosis of CRC and development of PC was 45.57 months (range, 9.46 to 119.50 months). There were one stage I PC, six stage II, one stage III, and five stage IV. The distribution of subsequent PC, by sex and age at initial CRC diagnosis is displayed in Table 2.

Table 3 shows the SIR of second primary PC following CRC, according to sex and age groups. Overall SIR was significantly higher in CRC patients compared to the general population (SIR, 14.44; 95\% CI, 12.71 to 16.16 ). An increased risk of subsequent PC was noted for both sexes: SIR 10.07 (95\% CI, 8.78 to 11.36) in men and 22.87 (95\% CI, 20.39 to 25.35) in women. However, the SIR of PC after CRC did not tend to increase linearly with aging.

To explore the risk factors in more detail, we performed additional analysis by stratifying by the following factors: age at 
Table 2. Clinical Features of Patients with Colorectal Cancer Who Did or Did Not Subsequently Develop Pancreatic Cancer

\begin{tabular}{|c|c|c|c|}
\hline Variable & CRC patients with subsequent PC & CRC patients without $\mathrm{PC}$ & p-value \\
\hline No. of patients & 13 & 4,809 & \\
\hline Male sex & $7(53.8)$ & $2,974(61.8)$ & 0.576 \\
\hline Age, yr & $65.0(48.0-72.0)$ & $61.0(45.0-74.0)$ & 0.128 \\
\hline \multicolumn{4}{|l|}{ Age group, yr* } \\
\hline $45-54$ & $1(7.7)$ & $1,152(24.0)$ & \\
\hline $55-64$ & $4(30.8)$ & $1,958(40.7)$ & \\
\hline $65-74$ & $8(61.5)$ & $1,699(35.3)$ & \\
\hline $\mathrm{BMI} \geq 25 \mathrm{~kg} / \mathrm{m}^{2}$ & $3(23.1)$ & $1,286 / 4,649(27.7)^{\dagger}$ & $>0.999$ \\
\hline Diabetes mellitus & $4(30.8)$ & $666 / 4,754(14.0)^{\ddagger}$ & 0.098 \\
\hline Smoking history & & & 0.296 \\
\hline Smoker or ex-smoker & $4(30.8)$ & $918 / 4,722(19.4)$ & \\
\hline Never smoker & $9(69.2)$ & $3,804 / 4,722(80.6)^{\S}$ & \\
\hline Chemotherapy for CRC & $10(76.9)$ & $3,533 / 4,598(76.8)^{\| \prime}$ & $>0.999$ \\
\hline Time to diagnosis of $\mathrm{PC}$, mo & $45.57(9.46-119.50)$ & - & \\
\hline
\end{tabular}

Data are presented as number (\%) or median (range).

CRC, colorectal cancer; PC, pancreatic cancer; BMI, body mass index.

*The age at the diagnosis of colorectal cancer was divided into 10-year groups; ${ }^{\dagger}$ We excluded 160 patients whose BMI was not identified in this analysis; ${ }^{\ddagger}$ We excluded 55 patients whose DM history was not identified in this analysis; ${ }^{8}$ We excluded 87 patients who did not answer the smoking history in this analysis; "We excluded 211 patients whose chemotherapy history was not identified in this analysis.

Table 3. SIR of Second Primary Pancreatic Cancer after Colorectal Cancer, Stratified by Age

\begin{tabular}{|c|c|c|c|c|}
\hline Age at initial diagnosis & Observed no.* & Expected no. ${ }^{\dagger}$ & SIR & $95 \% \mathrm{CI}$ \\
\hline \multicolumn{5}{|l|}{ Male sex, yr } \\
\hline $45-54$ & 152.21 & 8.70 & 17.50 & $14.72-20.27$ \\
\hline $55-64$ & 163.00 & 26.54 & 6.14 & $5.20-7.08$ \\
\hline $65-74$ & 364.63 & 58.49 & 6.23 & $5.59-6.87$ \\
\hline Total & 234.82 & 23.32 & 10.07 & $8.78-11.36$ \\
\hline \multicolumn{5}{|l|}{ Female sex, yr } \\
\hline $45-54$ & 0 & 4.18 & 0 & - \\
\hline $55-64$ & 272.11 & 13.44 & 20.25 & $17.84-22.65$ \\
\hline $65-74$ & 655.74 & 36.4 & 18.01 & $16.64-19.39$ \\
\hline Total & 325.91 & 14.25 & 22.87 & $20.39-25.35$ \\
\hline \multicolumn{5}{|l|}{ All patients, yr } \\
\hline $45-54$ & 86.73 & 6.47 & 13.40 & $10.58-16.23$ \\
\hline $55-64$ & 203.87 & 19.87 & 10.26 & $8.85-11.67$ \\
\hline $65-74$ & 468.66 & 46.12 & 10.16 & $9.24-11.08$ \\
\hline Total & 269.60 & 18.68 & 14.44 & $12.71-16.16$ \\
\hline
\end{tabular}

SIR, standardized incidence ratio; CI, confidence interval.

*The observed incidence of second primary pancreatic cancer (PC) in patients with colorectal cancer (CRC) was standardized using a population with CRC from the Korean Central Cancer Registry (KCCR) of the National Cancer Center; ${ }^{\dagger}$ The expected incidence rate of PC was obtained from the KCCR.

initial diagnosis of CRC, sex, DM, smoking, BMI, and history of chemotherapy for CRC (Table 4). We found no significant factor for increasing the risk of subsequent PC.

\section{DISCUSSION}

With the general adoption of screening colonoscopy and the progress in chemotherapy and surgical techniques, the 5-year survival rate of CRC has increased to 65\%. ${ }^{8}$ As a result, 
Table 4. Relative Risks of Subsequently Developing Pancreatic Cancer According to Age, Sex, Smoking, DM, BMI, and Prior Chemotherapy

\begin{tabular}{lclc}
\hline & Adjusted OR & \multicolumn{1}{c}{$95 \%$ CI } & p-value \\
\hline DM & 2.647 & $0.799-8.777$ & 0.111 \\
Age at diagnosis of CRC & 1.058 & $0.979-1.144$ & 0.156 \\
Smoking & 2.724 & $0.687-10.801$ & 0.154 \\
BMI $\geq 25 \mathrm{~kg} / \mathrm{m}^{2}$ & 0.729 & $0.199-2.671$ & 0.634 \\
Chemotherapy for CRC & 1.063 & $0.290-3.902$ & 0.926 \\
Female sex & 2.265 & $0.630-8.146$ & 0.211 \\
\hline
\end{tabular}

DM, diabetes mellitus; BMI, body mass index; OR, odds ratio; CI, confidence interval; CRC, colorectal cancer.

CRC survivors have a higher chance of developing a second primary malignancy, especially cancers that have similar carcinogenic mechanisms as those of CRC. Additionally, there has been an increase in the number of reported incidences of other solid organ malignancies after CRC, such as prostate cancer in men; breast, uterus, and ovarian cancers in women; and small intestine cancers in both sexes. ${ }^{9}$ In this regard, we supposed that PC would likely develop in patients with CRC due to the overlapping genetic predisposition. However, since patients with PC represent only a minority of the total cancer population, there are discrepant reports of the risk of second primary PC after CRC, depending on the type of research. Data from the Surveillance, Epidemiology, and End Results registries showed that CRC survivors had a higher risk of subsequent pancreatic adenocarcinoma (SIR, 1.22; 95\% CI, 1.09 to 1.35 ). ${ }^{10}$ A nationwide epidemiologic study from Sweden reported that the risk of second primary PC in colon cancer patients increased, by 1.77fold in men (95\% CI, 1.42 to 2.19) and 1.34-fold in women (95\% CI, 1.02 to 1.72$).{ }^{11}$ However, the risk was not significant for rectal cancer (SIR, 0.94; 95\% CI, 0.64 to 1.32 in men; SIR, 1.09; 95\% CI, 0.70 to 1.62 in women) in the same study. On the other hand, a 50-year observational study conducted in Connecticut, United States showed that patients with rectal cancer had a reduced risk of PC. ${ }^{12}$ In an international multicenter study, Shen et al. ${ }^{13}$ reported that the risk of PC following CRC was as low as 0.9-fold (SIR, 0.88; 95\% CI, 0.82 to 0.94).

Results from our current study showed that patients with CRC had a very high risk of developing PC compared to the general population. The overall incidence of PC in CRC patients aged 45 to 74 years was 269.60 per 100,000 patients, which was significantly higher than the 18.68 per 100,000 people reported in the general population of the same age (SIR, 14.44; 95\% CI, 12.71 to 16.16$)$. The incidence of $P C$ in our study might be high compared to other studies, since we monitored our patients very closely and thoroughly conducted diagnosis of PC. In addition, our results reflect the current trend of growing incidences of PC in the Korean population. ${ }^{14}$ It is also possible that PC and CRC share a more common etiology in Korea compared to other countries. Although we tried to rule out the possibility of metastasis and recurrence of CRC at the pancreas by imaging and pathologic evaluation, metastases might not have been differentiated from primary PC in some cases. Further studies are needed to validate and explain the high risk of subsequent PC in Korea.

In spite of high incidence of subsequent PC in CRC patients, PC tended to be diagnosed at the early stage. There is no statistics of PC stage at diagnosis in Korean general population, but in the United States, about 37\% of PC was either resectable or locally advanced. ${ }^{15}$ In this study about $62 \%$ of patients had resectable or locally advanced PC. Awareness of cancer risk and regular follow up could play a role in this trend. Also, there also could be difference in cancer behavior between first and second PC. This result suggests that well planned surveillance would be beneficial for early detection and treatment of subsequent PC in CRC survivors, which would ultimately bring survival gain. More studies are needed to understand the characteristics of subsequent PC.

Risk factors for subsequent PC were analyzed by DM, sex, aging, smoking, obesity, and chemotherapy history for CRC. However, we could not identify any significant risk factor for increasing subsequent PC. DM has previously been suggested to be a risk factor for PC in other studies. ${ }^{16,17}$ In our study, CRC patients with DM showed higher adjusted odds ratio (AOR) of 2.647 for subsequent PC, but there was no statistical significance (95\% CI, 0.799 to 8.777). As in most adult tumors, the incidence of PC is age-dependent, ${ }^{18}$ but increased age showed only a slight increased risk without statistical significance (AOR, 1.058; 95\% CI, 0.979 to 1.144 ). The SIR tended to be higher in women (SIR, 22.87) than in men (SIR, 10.07), but the sexual difference was not significant $(\mathrm{p}=0.211)$. Although other factors such as smoking, alcohol drinking, BMI, and previous chemotherapy have been suggested as risk factors for other types of cancer, these variables were not significant in our study. In previous studies, an increased risk of PC was observed in patients with familial adenomatous polyposis syndrome/Gardner's syndrome compared to the general population. ${ }^{19}$ Since cancer syndromes and familial PC are very rarely reported in Korea, there was a minimal relationship between familial PC or cancer syndromes and patients with subsequent PC in our registry.

This study has several limitations regarding generalization of the results. First, although the data were obtained prospectively, our analyses were performed retrospectively. Such investigational design may have presented some inherent drawbacks that could affect the results. For example, genetic abnormalities related to CRC and PC could not be identified. This information would have been very useful for determining the effects of genetic factors. Second, since our study was based on a single cancer registry, only a few PCs accumulated and the data was limited for providing reliable and robust results. Third, our inclusion of only histologically confirmed cases of PC in the anal- 
ysis may have caused underestimation of the disease. The loss of CRC patients to follow-up is also a point to be considered. For more reliable results, either a nationwide or an international multicenter study from high-quality cancer registries should be organized, and these databases should be combined to investigate relative risks and factors associated with PC.

In conclusion, we found that CRC survivors are relatively at a higher risk for second primary PC compared to the general population in Korea. In order to determine how often the patients should be screened and the optimal modality for screening, we recommend a nationwide CRC cohort study to be initiated and followed for the lifetime of patients, in which researchers collect and analyze detailed data including the patients' genetic abnormalities, family history, and medical history.

\section{CONFLICTS OF INTEREST}

No potential conflict of interest relevant to this article was reported.

\section{REFERENCES}

1. Shaib YH, Davila JA, El-Serag HB. The epidemiology of pancreatic cancer in the United States: changes below the surface. Aliment Pharmacol Ther 2006;24:87-94.

2. Zubarik R, Gordon SR, Lidofsky SD, et al. Screening for pancreatic cancer in a high-risk population with serum CA 19-9 and targeted EUS: a feasibility study. Gastrointest Endosc 2011;74:87-95.

3. Conroy T, Desseigne F, Ychou M, et al. FOLFIRINOX versus gemcitabine for metastatic pancreatic cancer. N Engl J Med 2011;364:1817-1825.

4. Von Hoff DD, Ervin T, Arena FP, et al. Increased survival in pancreatic cancer with nab-paclitaxel plus gemcitabine. N Engl J Med 2013;369:1691-1703.

5. Neugut AI, Robinson E. Multiple primary neoplasms. Cancer J 1992;5:245-248.

6. Jung KW, Park S, Kong HJ, et al. Cancer statistics in Korea: incidence, mortality, survival, and prevalence in 2008. Cancer Res
Treat 2011;43:1-11.

7. Breslow NE, Day NE. Statistical methods in cancer research. Volume II: the design and analysis of cohort studies. IARC Sci Publ 1987;(82):1-406.

8. Siegel R, Naishadham D, Jemal A. Cancer statistics, 2012. CA Cancer J Clin 2012;62:10-29.

9. McCredie M, Macfarlane GJ, Bell J, Coates M. Second primary cancers after cancers of the colon and rectum in New South Wales, Australia, 1972-1991. Cancer Epidemiol Biomarkers Prev 1997;6:155-160.

10. Rahimi E, Batra S, Thosani N, Singh H, Guha S. Increased incidence of second primary pancreatic cancer in patients with prior colorectal cancer: a population-based US study. Dig Dis Sci 2016;61:1652-1660.

11. Hemminki K, Li X. Familial and second primary pancreatic cancers: a nationwide epidemiologic study from Sweden. Int J Cancer 2003;103:525-530.

12. Hoar SK, Wilson J, Blot WJ, McLaughlin JK, Winn DM, Kantor AF. Second cancer following cancer of the digestive system in Connecticut, 1935-82. Natl Cancer Inst Monogr 1985;68:49-82.

13. Shen M, Boffetta P, Olsen JH, et al. A pooled analysis of second primary pancreatic cancer. Am J Epidemiol 2006;163:502-511.

14. Oh CM, Won YJ, Jung KW, et al. Cancer statistics in Korea: incidence, mortality, survival, and prevalence in 2013. Cancer Res Treat 2016;48:436-450.

15. Siegel RL, Miller KD, Jemal A. Cancer statistics, 2016. CA Cancer J Clin 2016;66:7-30

16. Everhart J, Wright D. Diabetes mellitus as a risk factor for pancreatic cancer: a meta-analysis. JAMA 1995;273:1605-1609.

17. Li D, Tang H, Hassan MM, Holly EA, Bracci PM, Silverman DT. Diabetes and risk of pancreatic cancer: a pooled analysis of three large case-control studies. Cancer Causes Control 2011;22:189197.

18. Maisonneuve P, Lowenfels AB. Epidemiology of pancreatic cancer: an update. Dig Dis 2010;28:645-656.

19. Giardiello FM, Offerhaus GJ, Lee DH, et al. Increased risk of thyroid and pancreatic carcinoma in familial adenomatous polyposis. Gut 1993;34:1394-1396. 